\title{
Classification of normal and abnormal patterns in medical thermography for the screening of breast cancer
}

\author{
by B.Vigneshwaran*, M. Sundaram*, M.Menaka\#, B.Venkatraman ${ }^{\# \#}$, D.N.Sangeeta ${ }^{\# \# \#}$, K.Malathy*** \\ * PG Student, Kamaraj College of Engineering and Technology, Virudhunagar, India \\ ${ }^{* *}$ Professor, Kamaraj College of Engineering and Technology, Virudhunagar, India \\ \# Scientific Officer, Quality Assurance Division, IGCAR, Kalpakkam, India \\ \#\# Associate Director, Radiological Safety and Environmental Group, IGCAR, Kalpakkam, India \\ \#\#\# Technical officer, Radiological Safety Division, IGCAR, Kalpakkam, India \\ *** Professor, Barnard Institute of Radiology, MMC, Chennai, India
}

\begin{abstract}
Breast cancer is one of the most important causes of death among women. Breast cancer can be treated effectively only if it is detected at the early stages. Thermography plays a vital role in early detection of breast cancer. The principal aim of early detection of breast cancer is to identify the disease at a more curable stage and thus improve the prognosis and other vital clinical outcomes. In this work, we propose a classification scheme to classify the breast tissues as normal, benign or malignant and thermograms were taken from women of various age groups and various breast diseases. The proposed system consists of three stages. In the first stage pre-processing and segmentation of region of interest is performed. In the second stage, feature extraction matrix is generated using GLCM and all the detailed coefficients from 2D-DWT of a thermogram. The final stage is to classify the breast tissues with the help of Support Vector Machine (SVM), K-Nearest Neighbor (KNN). The proposed approach is applied to set of 35 images of various patients (normal, benign, malignant) and classification accuracy of $95.71 \%$, sensitivity of $100 \%$, specificity of $88.5 \%$, Positive Prediction Value (PPV) of $93.62 \%$ and Negative Prediction Value (NPV) of $100 \%$ is achieved.
\end{abstract}

\section{Introduction}

Thermography dates back to the 1800s; the first 'heat picture" was produced in 1840 by Sir John Herschel. In the early 1900s, infrared research was classified by the military and unavailable for medical application. It was not until 1956 that technical information on thermography was declassified, and in 1961 the first images were used in medical research to confirm that skin temperatures were elevated on the surface of the breast over a cancer Thermography is a non-invasive and non-radiating analysis tool for analyzing physiological functions related to the control of skin-temperature. This rapidly developing technology is used to detect and locate thermal abnormalities characterized by an increase or decrease found at the skin surface. The technique involves the detection of infrared radiation that can be directly correlated with the temperature distribution of a defined body region. It is an imaging method which can diagnose cancer and other pathologies by analysis of tissue surface temperature as was obtained by an IR camera. Most of the researches and the clinical applications of thermography are associated with early detection of breast cancer. 
This use was first suggested as early as 1956 and is based on the fact that malignant tumors produce a local increase at the skin surface as a result of an increased angiogenesis, increased perfusion and increased metabolism in and around an embedded malignant tumor. Accordingly, thermography is considered today as a complimentary screening tool rather than a first line screening modality. According to the American Cancer Society, "Thermography has been around for many years, and some scientists are still trying to improve the technology to use it in breast imaging. But no study has ever shown that it is an effective screening tool for finding breast cancer early. In addition to breast cancer diagnosis, thermography was also suggested as a diagnostic tool for various other disorders, including: colon cancer, occlusive vascular disorders, neural disorders and ophthalmology.

\section{Theory}

Breast cancer is the most commonly diagnosed cancer in female subjects accounting for about $30 \%$ cancers. The average age for death due to breast cancer was 68 years. Approximately $0.0 \%, 0.9 \%, 6.0 \%, 15.0 \%$, $20.8 \%, 19.7 \%, 22.6 \%$ and $15.1 \%$ deaths occurred between the age groups of under $20,20-34,35-44,45-54,55-$ $64,65-74,75-84$ and above 85 years respectively. It has been reported that $0 \%$ diagnosis was possible under age of 20 years, whereas $1.9 \%, 10.2 \%, 22.6 \%, 24.4 \%, 19.7 \%, 15.5 \%$ and $5.6 \%$ diagnosis were possible within the age groups of $20-34,34-44,45-54,55-64,65-74,75-84$ and above 85 years respectively. Studies show that, early detection can lead to $85 \%$ survival chance compared to $10 \%$ for late detection. Therefore, early detection is the key factor for successful breast cancer treatments. Food and Drug Administration (FDA) approved thermography as an adjunctive tool for diagnosis of breast cancer. Thermography provides additional functional information on the thermal and vascular condition of the tissues. It is non-invasive breast tumors detection modality in which abnormal breast thermogram indicates significant biological risk. Tumors generally have an increased blood supply and an increased metabolic rate which leads to localized high temperature spots over such areas, rendering them to be visualized by thermography. Apart from passive breast imaging, cold stimulation based imaging procedures are also in practice.

Blood vessels, produced by cancerous tumors are simple endothelial tubes devoid of a muscular layer. Such blood vessels fail to constrict in response to sympathetic stimulus like a sudden cold stress and show a hyperthermic pattern due to vasodilatation. Deng and Liu showed that, induced evaporation enhances thermographic contrast in case of tumors underneath the skin. They used water and $75 \%$ medical ethanol solution as evaporant, which was sprayed over the skin surface and then images were acquired. Their study shows an improved diagnostic accuracy especially in the early stages of deeply embedded tumors. It has been confirmed that abnormal patterns in the infrared images are the highest risk indicators of breast cancer development in future. Gamagami studied angiogenesis by thermography and reported that in 15\% cases thermography could detect cancers which were not discernible by mammography. He had also shown that, hypervascularity and hyperthermia were visible in $86 \%$ cases of non-palpable breast cancers. The growth rate related prognostic indicators are strongly associated with the thermograms. Thermovascular activities in breast are directly related to prognosis and aggressiveness of the tumor. It has been reported that, hot cancers (most active cancer cells) show a poorer prognosis with $24 \%$ survival rate in 3 years, whereas for cooler cancers prognosis is much better with $80 \%$ survival rate. Apart from Thermography many other techniques are available for breast imaging, like mammography, MRI, CT, ultrasound, etc. Mammography is the most popular technique, but suffers from a few inherent problems, like exposure to X-ray radiation, relatively higher cost, problem in detecting tumors of smaller sizes and low sensitivity for firm breasts of younger women. Thermography does not suffer from such problems and hence, it is a novel tool for investigation of breast cancer. 
Thermography is an effective alternative diagnosis tool, especially in female subjects with dense breast parenchyma. The IR images of the tumor and the surrounding normal cortex regions show that, the former is at a higher temperature compared to the later.

\section{Materials and Methods}

\subsection{Thermal camera}

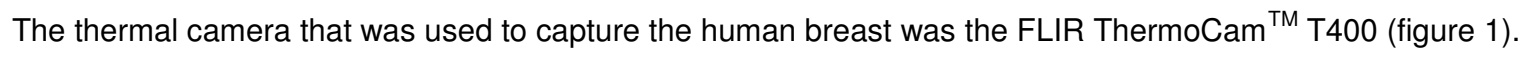
The camera detector array is a focal plane array (FPA) uncooled micro bolometer at the size of $320 \times 240$ pixels. The camera is working at the spectral range of 7.5 to $13 \mu \mathrm{m}$. The camera is capable of measuring temperature between $-20^{\circ} \mathrm{C}$ and $+120^{\circ} \mathrm{C}$ with thermal sensitivity of $50 \mathrm{mK}$, thermal accuracy of $\pm 2 \%$ and frame rate of 60 frames per second.

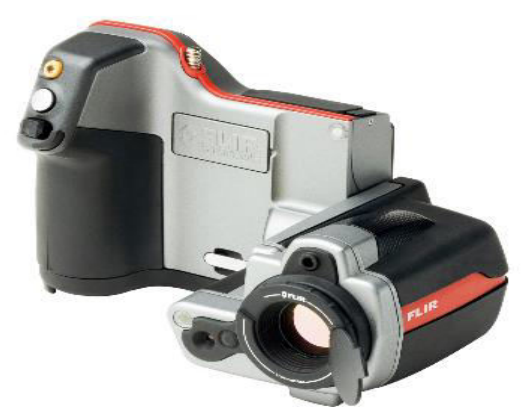

Figure 1. Thermal camera

\subsection{Proposed Method}

In this research, we used five techniques, which come under the task of image processing which are as follows

1. Image Acquisition.

2. Image segmentation.

3. Image Pre-Processing.

4. Feature Extraction.

5. Image Classification.

\subsubsection{Image Acquisition}

Image Acquisition is the process of retrieving an image from sources such as camera, computers etc. In case of medical image processing the source of image is from digital computer for Mammography and thermography. This is the first step in image processing. The camera that was used to capture the human breast was the FLIR ThermoCam $^{\mathrm{TM}}$ T400.Breast thermograms of a 24 normal, 23 benign and 23 malignant patients are taken for the analysis. 


\subsubsection{Image segmentation.}

Image segmentation is the processes of sub dividing an image into its constituent region. There are several methods to perform image segmentation. Edge method is based on detection of edges in order to identify the boundary between regions. Some of the edge detection operators are sobel, prewit and canny. Region method is based on assigning a pixel to a given region based on their degree of mutual similarity. Some of the region based methods are Region splitting, region merging, and region growing. In this work Image segmentation is done by selecting the region of interest (left and right breast) from the thermograms.

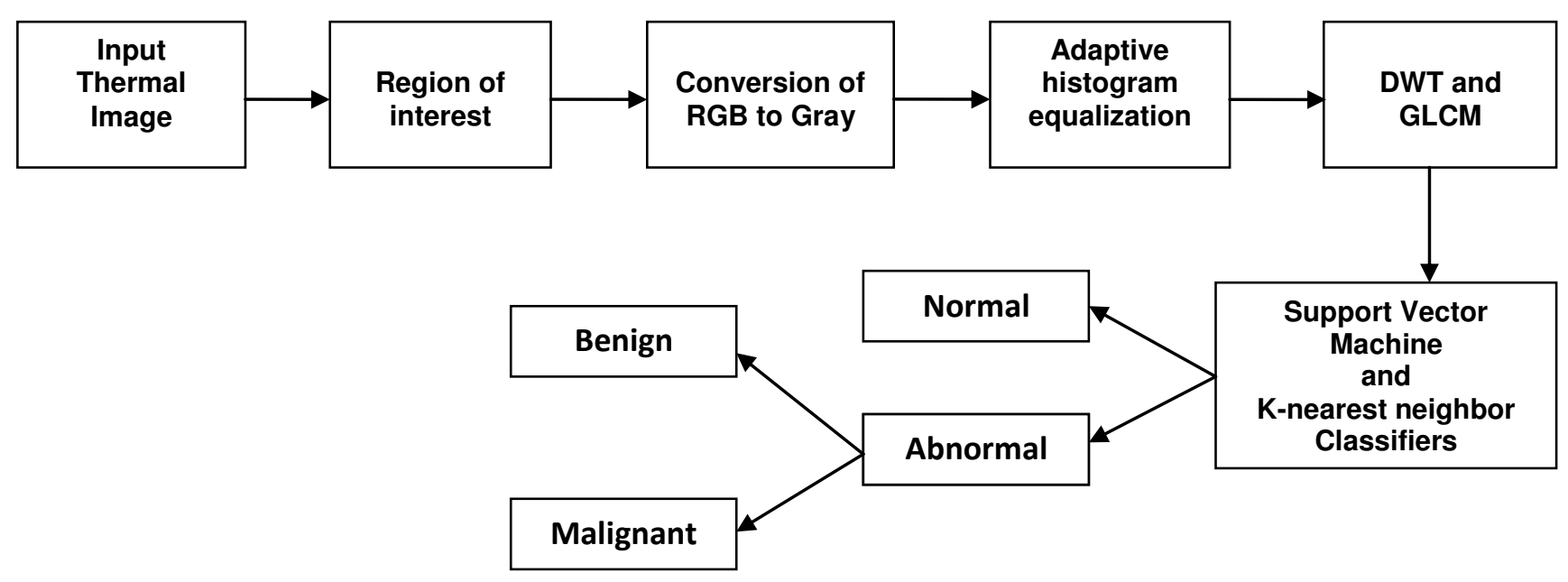

Figure 2: Block Diagram of Proposed Method

\subsubsection{Image Pre-Processing}

Preprocessing deals with the non-uniformity of IR images. Another source of non-uniformity is fluctuating lighting conditions such as incandescent bulb or direct sun light. Therefore, it may be required to resize the images using techniques, such as template and interpolation methods. Another source of non-uniformity is fluctuating lighting conditions. The adaptive histogram equalization method can eliminate the discontinuous background, which may result from discontinuous lighting or other inconsistencies that originate from the IR image capturing techniques. It maps the input to the output image pixel intensity values such that the output image exhibits uniformly distributed intensities. This increases the dynamic range of the image histogram. First step in Pre-processing in this work is to convert the RGB image to Gray image. Since, gray image provides more detailed information about the features than the RGB image this process is done. Next step in pre-processing is to apply adaptive histogram equalization to the resultant image obtained from first step. Adaptive Histogram equalization is a technique for improving the contrast in the image. It computes several histogram, each corresponding to a distinct region of an image and uses them to redistribute the intensity values of the image. AHE improves the contrast in the image by transforming each pixel value with a transformation function derived from the neighborhood pixel. It is an excellent contrast enhancement technique for both natural images and medical images 


\subsubsection{Feature Extraction}

In the field of Image processing and pattern recognition, the feature extraction is a technique used for collecting various features from image and dimensionality reduction. Here, we use two different feature extraction methods to extract the various features from each segmented thermal images.

1. Feature Extraction based on intensity-Wavelet transform

2. Feature extraction based on texture-Gray-Level Co-occurrence Matrix

The wavelet based features such as Kurtosis and Skewness are calculated in this work.

1. Kurtosis - It is a measure of how the image corresponding to either cancerous or noncancerous class, is peaked or flat relative to the probability distribution. The image with high kurtosis tends to have different peak near mean value and decline rapidly. Similarly the image having low kurtosis tends to have flat top near mean rather than sharp peak. The kurtosis of a distribution can be calculated as:

$$
\operatorname{Kurtosis}(\mathrm{Ku})=\frac{\sum_{r_{k}=1}^{L} \mathrm{P}\left(\mathrm{r}_{\mathrm{k}}\right) \times\left(r_{k}-\mu\right)^{4}}{\sigma^{4}}
$$

Where $\mu$ is the mean and $\sigma$ is the standard deviation of the image with gray vector varies from 1 to $L$

2. Skewness - It is a measure of the asymmetry of the pixel value of image around the sample mean. If Skewness is negative, the pixels in the image are spread out more to the left of the mean than to the right. Similarly, if Skewness is positive, the pixels in the image are spread out more to the right. The Skewness of the normal distribution (or any perfectly symmetric distribution) is zero. The Skewness is defined mathematically as:

$$
\operatorname{Skewness}(\mathrm{S})=\frac{\sum_{r_{k}=1}^{L} \mathrm{P}\left(\mathrm{r}_{\mathrm{k}}\right) \times\left(r_{k}-\mu\right)^{3}}{\sigma^{3}}
$$

Where $\mu$ is the mean and $\sigma$ is the standard deviation of the image with gray vector varies from 1 to $L$

GLCM based texture features such as Contrast, Correlation and Inverse difference moment are calculated in this work.

3. Contrast- It is the measure of local intensity variation and how the grey levels vary in the image and to what extent their distribution is biased to black or white.

$$
\text { Contrast }=\sum_{n=0}^{\mathrm{G}-1} \mathrm{n}^{2}\left\{\sum_{\mathrm{i}=1}^{\mathrm{G}} \sum_{\mathrm{j}=1}^{\mathrm{G}} \mathrm{P}(\mathrm{i}, \mathrm{j})\right\},|\mathrm{i}-\mathrm{j}|=\mathrm{n}
$$


4. Inverse Difference Moment: It is influenced by the homogeneity of the image. Because of the weighting factor $1 / 1+(i-j)^{2}$ IDM will get small contributions from in homogeneous areas. The result is a low IDM value for inhomogeneous images, and a relatively higher value for homogeneous images.

$$
\operatorname{IDM}=\sum_{\mathrm{i}=1}^{\mathrm{G}} \sum_{\mathrm{j}=1}^{\mathrm{G}} \frac{1}{1+(i-j)^{2}} \mathrm{P}(\mathrm{i}, \mathrm{j})
$$

5. Correlation-It is a measure of gray level linear dependence between the pixels at the specified positions relative to each other.

$$
\text { correlation }=\sum_{i=0}^{G-1} \sum_{i=0}^{G-1} \frac{\{\mathrm{ix} j\} \times P\left(i_{i} j\right)-\{\mu(x) \times \mu(y)\}}{\sigma(x) \times \sigma(y)}
$$

\subsubsection{Classification - Machine learning}

Machine learning is a multidisciplinary field of study that mainly concerned with the design of algorithms which allow computers to learn. The term "Machine Learning" comes from the artificial intelligence community but now a day it mainly the focusing area for many branches of engineering and science. Learning mainly refers to learning from data or feature set. There are different learning methods for statistical data analysis. In this study, thermograms are first classified as Normal and Abnormal and further classifying abnormal as benign or malignant. KNN and SVM are used to classify the images

\section{Results and Discussion}

In this work, the camera that was used to capture the human breast was the FLIR ThermoCam ${ }^{\text {TM }}$ T400.Breast thermograms of a 24 normal, 23 benign and 23 malignant patients are taken for the analysis and few images are shown below.
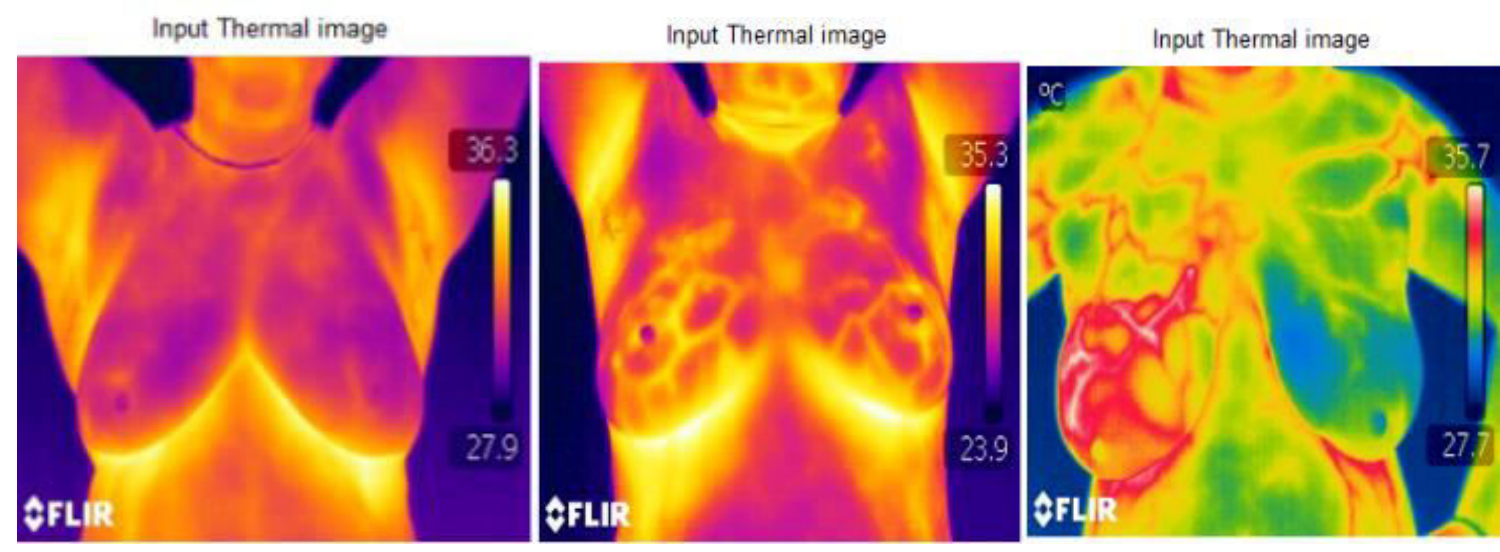

Figure 3: Input Thermal Images: From Top Left IR-1221, IR-0214,IR-2116 
The thermal breast images are segmented by cropping the region of interest from the input thermal image. The segmented left and right breast images are then processed for detecting whether the breast is affected by cancer or not.

night breast

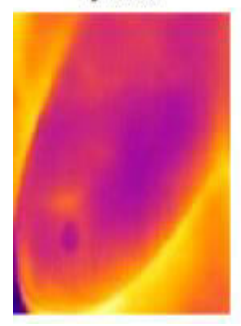

left breast

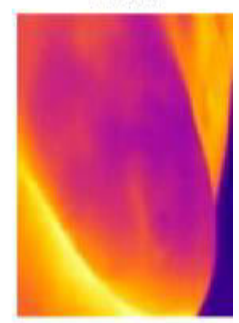

right breast

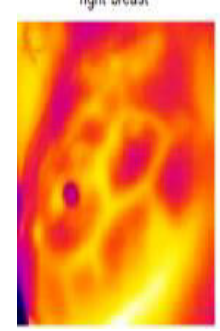

left breast

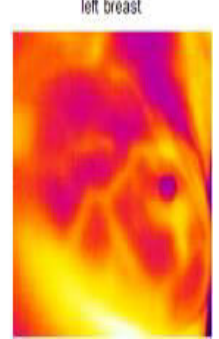

right breast

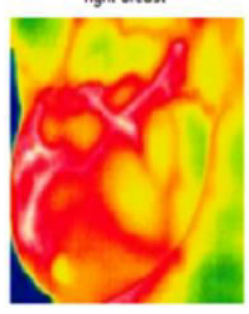

lett breast

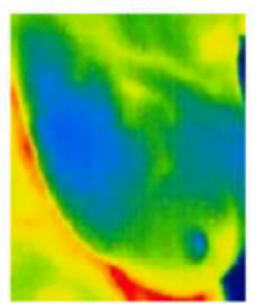

Figure 4: Segmented Right and Left Breast for the input images

Next step involved in proposed method is to convert the thermal image (RGB image) into gray scale image. This is process is done because Gray scale image has the ability of providing more detailed information about the features present in an image than the RGB image.
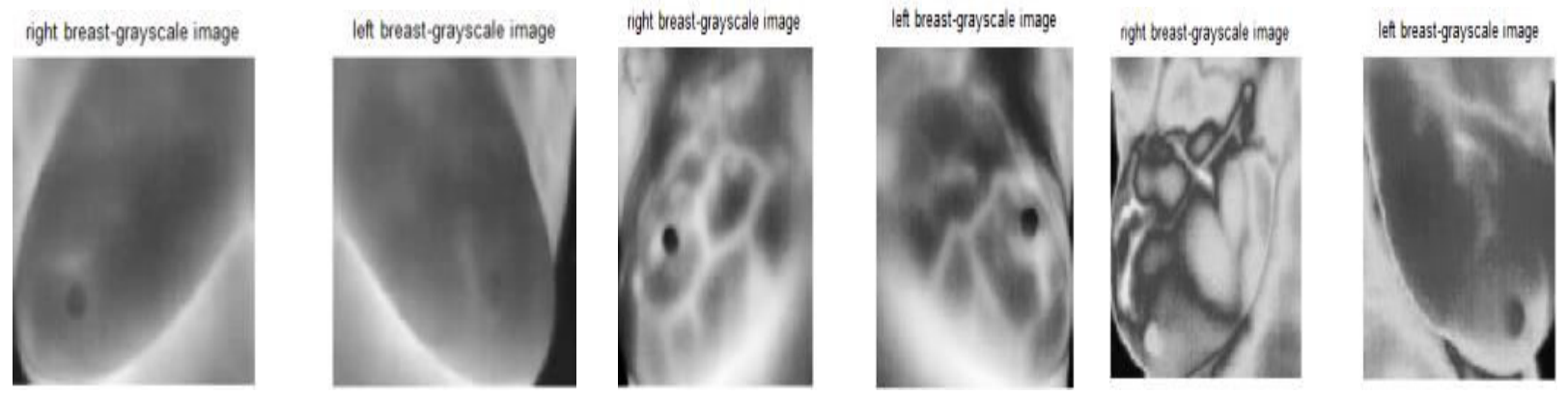

Figure 5: Gray-Scale Image of Right and Left Breast

Next step in pre-processing is to apply adaptive histogram equalization to the resultant image obtained from first step. Adaptive Histogram equalization is a technique for improving the contrast in the image.
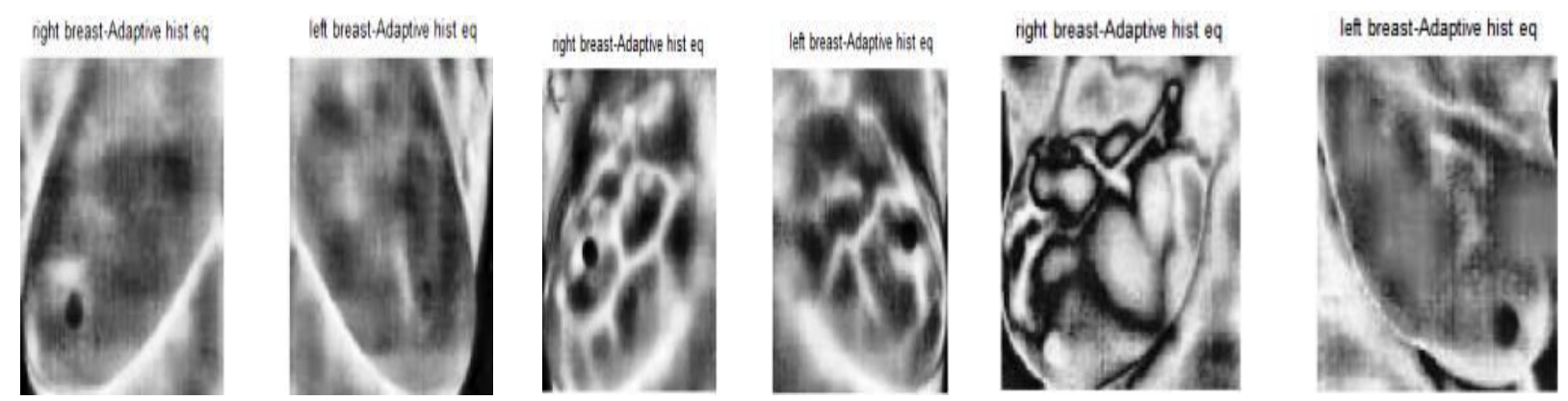

Figure 6: Image of Right ond Left Breast After Applying Adaptive Histogram Equalization 


\subsection{Classification Parameters and Results}

In this work, normal and abnormal images are classified with the help of five features (2 from 2D-DWT and 3 from GLCM).There are Kurtosis, Skewness, Contrast, Inverse difference moment, Correlation
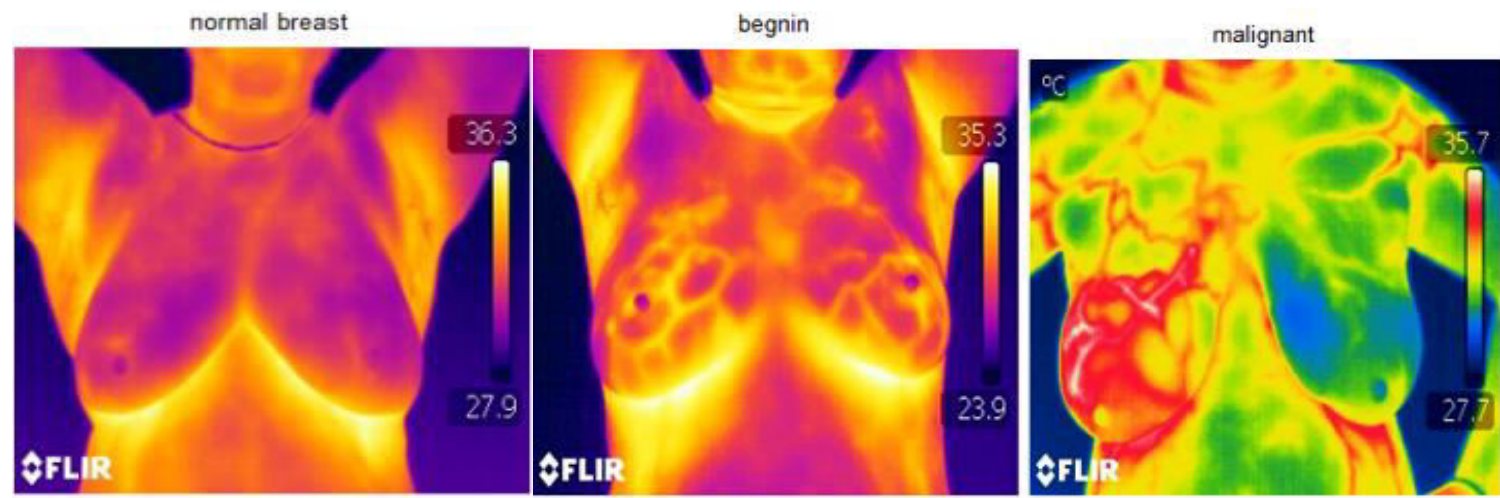

Figure 7: Classification Results Corresponding to the Input Images

Table 1: Confusion Matrix for K-NN and SVM Classifiers

\begin{tabular}{|c|c|c|}
\hline TRUE & Cancerous & Noncancerous \\
\hline Cancerous & $44(\mathrm{TP})$ & $0(\mathrm{FN})$ \\
\hline Noncancerous & $3(\mathrm{FP})$ & $23(\mathrm{TN})$ \\
\hline
\end{tabular}

Table 2: Comparisons of Performance Measures

\begin{tabular}{|c|c|c|c|c|}
\hline Authors & Classifier used & Accuracy & Sensitivity & Specificity \\
\hline Ng and Fok & ANN & $61.5 \%$ & $68.9 \%$ & $40 \%$ \\
\hline Borchaett et al & SVM & $85.7 \%$ & $95.8 \%$ & $88.2 \%$ \\
\hline Ng et al & RBF networks & $80.9 \%$ & $81.2 \%$ & $90.4 \%$ \\
\hline Archarya et al & SVM & $88.1 \%$ & $85.7 \%$ & $88.2 \%$ \\
\hline $\begin{array}{c}\text { Ng and Kee } \\
\text { RBF networks }\end{array}$ & $80.9 \%$ & $91.7 \%$ & $25 \%$ \\
\hline $\begin{array}{c}\text { Resmini } \\
\text { Our proposed } \\
\text { method }\end{array}$ & K-NN and SVM & $95.71 \%$ & $100 \%$ & $88.5 \%$ \\
\hline
\end{tabular}




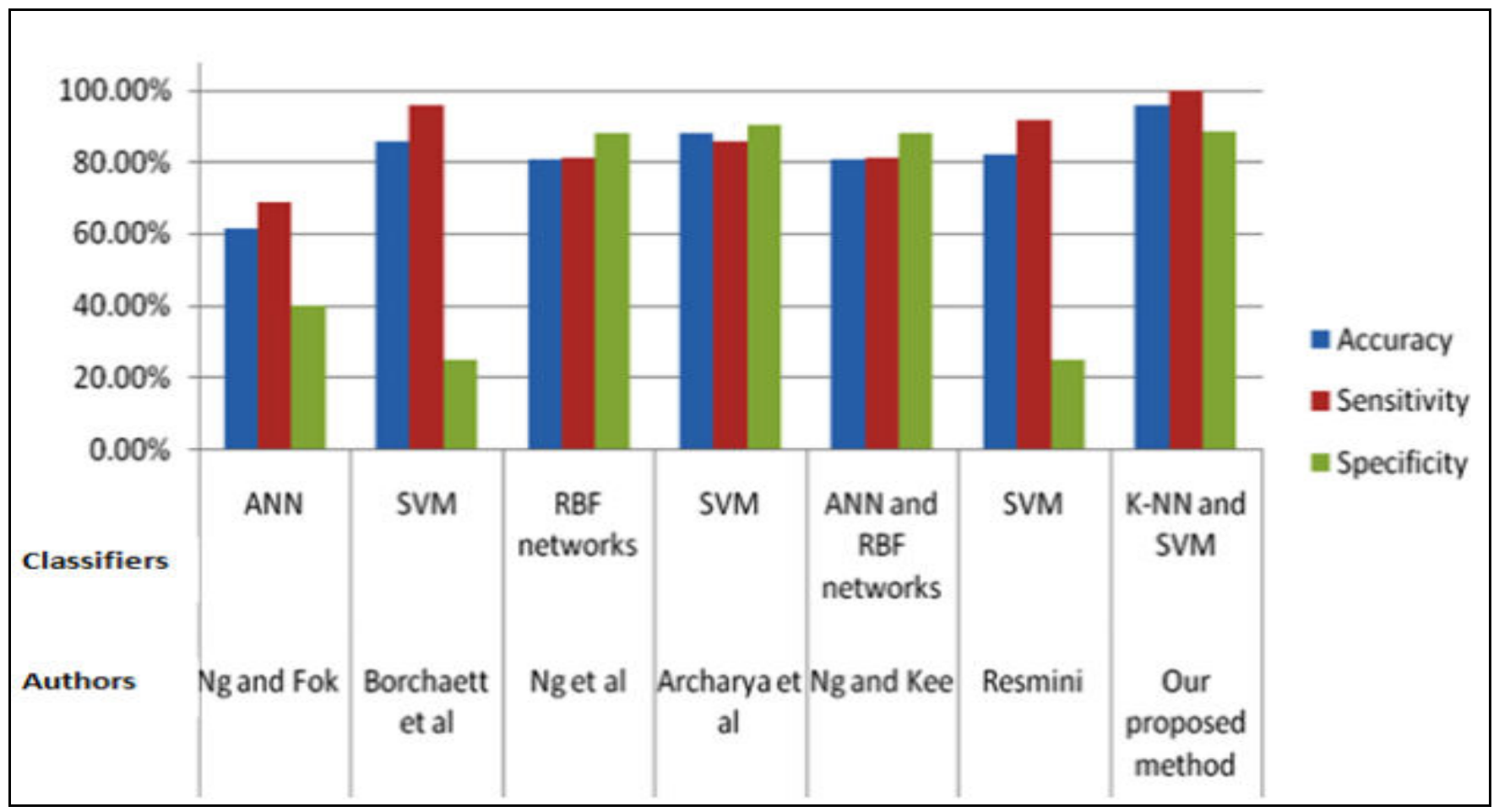

Figure 8: Comparisons of Performance Measures

\section{Conclusion}

Early screening plays a pivotal role in management of breast cancer. Any kind of abnormality or diseases can be cured easily if it is detected earlier. Breast thermograms are processed to extract features based on intensity and texture from both breasts to classify them as malignant, benign and normal classes. Medical Thermography has shown promising results in this work with accuracy of $95.71 \%$, sensitivity of $100 \%$, specificity of $88.5 \%$, positive predictive value $93.62 \%$ and negative predictive value of $100 \%$. Being a non-contact, pain free, radiation free procedure it received a good patient response and high accept ability. From this study, it is concluded that Medical Thermography is well suited as a screening tool and its use in combination with other laboratory and outcome assessment tools, can lead to significant improvement in the management of breast cancer in India. This can be used to improve the current scenario in India where because of late diagnosis considerable amount of patients suffering from breast cancer suffer death. Moreover it can lead to easy and acceptable screening in rural areas also where mammography and biopsy studies are still a distant dream. Breast cancer is a highly treatable disease, with $97 \%$ chance of survival if discovered early. Hence by screening the human breast using thermography will increase the survival rate of the patients affected by breast cancer in India. 


\section{REFERENCES}

[1] Akay MF (2009), 'Support Vector Machines Combined With Feature Selection For Breast Cancer Diagnosis'. Expert Systems with Applications 36(2, Part 2):3240-3247.

[2] Arodź T, Kurdziel M, Sevre EOD, Yuen DA (2005), ' Pattern recognition techniques for automatic detection of suspicious-looking anomalies in mammograms'. Computer Methods and Programs in Biomedicine 79(2):135-149.association rules and neural network. Expert Syst Appl 36(2):3465-3469 doi:10.1016/j.eswa.2008.02.064

[3] B.B. Lahiri, S. Bagavathiappan, T. Jayakumar, John Philip, 'Medical applications of infrared thermography: A review', Elsevier- Infrared Physics \& Technology 55 (2012) 221-235. Computer Vision and Image Analysis 8(2):39-50.

[4] Jele U, Fevens T, Krzy A (2008), 'Classification of Breast Cancer Malignancy Using Cytological Images of Fine Needle Aspiration Biopsies'. Int J Appl Math Computer Science 18(1):75-83.

[5] Karabatak M, Ince MC (2009, 'An expert system for detection of breast cancer based on association rules and neural network'. Expert Syst Appl 36(2):3465-3469 doi:10.1016/j.eswa.2008.02.064.

[6] L.A.Bezerra, M.M.Oliveira , T.L.Rolim , A.Conci "Estimation of breast tumor thermal properties using infrared images" Elsevier-Signal Processing 93 (2013) 2851-2863.

[7] M. Kontos, R. Wilson, I. Fentiman, 'Digital infrared thermal imaging (DITI) of breast lesions: sensitivity and specificity of detection of primary breast cancers', Elsevier-Clinical Radiology 66 (2011) 536-539.

[8] Mahnaz Etehadtavakol, Vinod Chandran, Hossien Rabban, 'Discrete Wavelet Transform Based Texture Features and Image Classification Of Breast Thermograms', Elsevier-Infrared Physics \& Technology 61 (2013) 274-286.

[9] Mahnaz Etehadtavakol, Vinod Chandran, E.Y.K. Ng, Raheleh Kafieh, 'Breast cancer detection from thermal images using bispectral invariant features' Elsevier-International Journal of Thermal Sciences 69 (2013) 21 36

[10] Martins LDO, Junior GB, Silva AC, Paiva ACD, Gattass M (2009) Detection of Masses in Digital Mammograms using K-means and Support Vector Machine. Electronic Letters on Computer Vision and Image Analysis 8(2):39-50.

[11] Medical Infrared Imaging Principles and Practices” By Mary Diakides, Joseph D.Bronzino, Donald Peterson.

[12] Muffazzal Rassiwala, Poonam Mathur, Raj Kumar Mathur, 'Evaluation of Digital Infra-Red Thermal Imaging as an Adjunctive Screening Method for Breast Carcinoma', Elsevier-International Journal of Surgery 12 (2014) 1439-1443.

[13] Oliver Faust, U.Rajendra Acharya, E.Y.K.Ng, Tan Jen Hong, 'Application of infrared thermography in computer aided diagnosis', Infrared Physics \& Technology 66 (2014) 160-175.

[14] Rejani YIA, Selvi ST (2009), 'Early detection of breast cancer using SVM classifier technique', International Journal on Computer Science and Engineering 1(3):127-130.

[15] Schaefer G, Závišek M, Nakashima T (2009), 'Thermography based breast cancer analysis using statistical features and fuzzy classification'. Pattern Recognition 42(6):1133-1137.

[16] Sheeja V Francis, M. Sasikala, G. Bhavani Bharathi, 'Breast Cancer Detection in Rotational Thermography Images Using Texture Features', Elsevier-Infrared Physics \& Technology 67 (2014) 490-496.

[17] Tiago B.Borchartt, Aura Concia, RitaC.F.Limab, Roger Resminia, 'Breast Thermography From An Image Processing Viewpoint: A Survey', Elsevier-Signal Processing 93 (2013) 2785-2803. 